\title{
Erratum: "Do Neutrino Oscillations Allow an Extra Phenomenological Parameter" [JETP Letters 73, no. 8, 380 (2001)]
}

\section{S. Tsukerman}

PACS numbers: 14.60.Pq; 12.15.Ff

In this paper, all references to formula numbers in the second column of the text are erroneous. One should read

(1) and (2) instead of (2) and (3),

(4)-(6) instead of (6)-(8),
(1) instead of (5),

(7) instead of (3),

(3) and (6) instead of (7) and (8).

The author and editorial board wish to apologize for this error. 